\title{
Abnormally differentiated bone marrow mesenchymal stem cells develop into tumors in rats
}

\author{
Hongxia Yuan ${ }^{1}$, Shiqi Liao ${ }^{1}$, Jiayu Zeng ${ }^{2}$, Xiaojuan Huang ${ }^{1}$, Jieyu Zhang ${ }^{1}$, Jing Zhao ${ }^{2}$,Yuxian Wang ${ }^{2}$, \\ Hong Zhang ${ }^{2}$, Xiaoqi Wang ${ }^{1}$, Shidong Liao ${ }^{1}$, Wenru Tang ${ }^{1}$, Zhengli Wei ${ }^{1}$, Caiping Tian ${ }^{1}$, Rong Yang ${ }^{1}$, \\ Fang Liu ${ }^{1}$, Hongyun Guo ${ }^{1}$, Yongdong Zhang ${ }^{1}$ \\ ${ }^{1}$ Laboratory of Molecular Biology, Institute of Gansu Medical Science Research, Lanzhou 730050, China; ${ }^{2}$ College of Life Sciences, Northwest \\ Normal University, Lanzhou 730070, China \\ Contributions: (I) Conception and design: S Liao, J Zeng, S Liao; (II) Administrative support: S Liao; (III) Provision of study materials or patients: H \\ Yuan, Z Wei, C Tian, H Guo, Y Zhang; (IV) Collection and assembly of data: X Huang, J Zhang, J Zhao, Y Wang, H Zhang, X Wang, W Tang; (V) \\ Data analysis and interpretation: H Yuan, S Liao, J Zeng, R Yang, F Liu; (VI) Manuscript writing: All authors; (VII) Final approval of manuscript: All \\ authors. \\ Correspondence to: Shiqi Liao. Gansu Academy of Medical Sciences, 2 Xiaoxihu E.S., Lanzhou 730050, China. Email: liaosq@nwnu.edu.cn; \\ Jiayu Zeng. College of Life Sciences, Northwest Normal University, 967 Anning East Road, Lanzhou 730070, China. Email: zengjy@nwnu.edu.cn.
}

Background: The generation of cancer stem cells is essential for the formation of tumor tissues; insightful understanding of the process may offer breakthroughs in tumor research and treatment. It is hypothesized that somatic stem cells (SSC) undergoing abnormal differentiation and evolution are able to form heterogeneous cell clusters, leading to onset of tumors.

Methods: Bone marrow mesenchymal stem cells (BMSCs) were isolated and cultured, then BMSCs were induced to differentiate into osteoblasts, adipocytes and neuroblasts. The differentiation process of BMSCs was interrupted at certain time points, and the cells at various stages of maturation were obtained. The resulting cells were further cultured to their fourth generation, then they were subject to the soft agar colony forming assay to screen the mutated cells that form clusters after losing their contact inhibition. The mutant cells were cultured for 5 days, then inoculated on the renal fascia of mice, which were pre-treated with immunosuppress reagent cyclophosphamide (CTX).

Results: Tissue biopsies confirmed a generation of heterogeneous cell clusters in the mice. These clusters morphologically resemble Hela cells, as they share similar pathophysiology, histomorphology and cell biology features with cancer tissues. These findings indicate that the evolved heterogeneous cell clusters can lead to cancer.

Conclusions: This study reveals the pathogenesis of tumors in respect to stem cells. Tumor stem cells may arise from the abnormal differentiation and evolution of SSC. From then on, tumor production evolves including heterogeneity, metastasis, immune escape and infinite increment.

Keywords: Tumor; bone marrow mesenchymal stem cells; heterogeneous cell clusters; abnormal differentiation; tumor evolution

Submitted Jun 07, 2018. Accepted for publication Nov 14, 2018.

doi: $10.21037 /$ tcr.2018.12.16

View this article at: http://dx.doi.org/10.21037/tcr.2018.12.16

\section{Introduction}

For the detection and treatment of cancer, it is very important to elucidate the mechanism governing the formation of cancerous tissues. As far back as 1976, Nowell first proposed that cancer is an evolutionary "organism" (1). In a previous study, Maley et al. comprehensively investigated Barret's esophagus (2). They confirmed that the transition from precancerous lesions to cancer was 
indeed an evolutionary process, with a cell population adapting to natural selection. This finding was confirmed in a study conducted by Anderson et al., which proved that the phenotype and morphology of tumors significantly changed when selective pressure was exerted on the tumor microenvironment (3). Furthermore, Merlo et al. defined cancer as a unique "ecosystem" and they explained the evolutionary nature of cancer progression (4). In summary, cancer can be considered as an "organism" that has evolved from a cell population in a unique microenvironment. The first evidence of a cancer stem cell was provided by Hamburger et al in 1977, who found that among the 1,0005,000 solid tumor cells, only one tumor cell could form cell clones in a soft agar (5). In 1997, acute leukemia stem cells were first successfully isolated by Bonnet and Dick (6). Later on, more cancer stem cells of specific immuno-phenotypes from chronic myeloid leukemia, glioma and breast cancer tissues were isolated (7-10). These studies have proven that cancer stem cells do exist in cancerous tissues.

Stem cells are early undifferentiated cells, and they have pluripotency and self-renewal capabilities (11). Under certain conditions, stem cells can differentiate into mature cells that perform different functions. A variety of tissues and organs can also be synthesized from stem cell culture. Therefore, stem cells are also called "universal cells" in the medical community. In the 1980s, Piersma et al. found that bone marrow stromal cells (BMSCs) differentiate into various interstitial tissues, such as bone, cartilage, fat and even muscle tissue $(12,13)$. In the 1970s, Potter et al. and Pierce et al. first proposed the hypothesis that cancer stem cells originated from normal stem cells that have undergone a maturation arrest $(14,15)$. We believe that cancer arises from the heterogeneous cell clusters, which gradually evolve after the differentiation of normal stem cells is interfered with in a microenvironment. In this study, rat BMSCs were selected as the model to explore this mechanism of cancer formation.

\section{Methods}

\section{Materials}

Six-week-old Wistar female rats weighing 150-160 g and Kunming female mice weighing about $22 \mathrm{~g}$ were purchased from the Institute of Laboratory Animal Sciences (CAMS and PUMC, Beijing, China). The experimental mice were maintained under specific pathogen-free (SPF) conditions at the Experimental Animal Center of Gansu University of
Traditional Chinese Medicine (TCM) (Lanzhou, China). In this experiment, we performed all the animal procedures in accordance with the Guide for Care and Use of Laboratory Animals. All the experimental protocols were approved by the Animal Ethics Committee of Gansu University of TCM (Lanzhou, China).

\section{Isolation and culture of rat BMSCs and Hela cells}

Typically, the isolation of rat BMSCs is followed by the method reported by Tropel et al. (16). Wistar rats were killed by cervical dislocation, under sterile conditions. The femurs and tibiae were removed and immersed in a $0.01 \mathrm{mmol} / \mathrm{L}$ phosphate buffer saline (PBS) solution and bone ends were exposed. A Dulbecco's modified eagle's medium (DMEM)/F12 (Hyclone, USA) medium was used to flush marrow until the bones turned pale. The resulting cell suspension was filtered with a 200-mesh sieve, cells were re-suspended in medium containing $10 \%$ fetal bovine serum (FBS) (Hyclone, USA) and 1\% L-Gln (Hyclone, USA). The cells were then inoculated into a cell culture dish and incubated at $37^{\circ} \mathrm{C}$ in a $5 \% \mathrm{CO}_{2}$ atmosphere. The culture medium was replaced once every three days, and the non-adherent cells were discarded. When the cells reached $70-80 \%$ confluency, they were passaged for the purification process. Then the pure BMSCs were stored in liquid nitrogen.

Frozen Hela cells were resuscitated and cultured in DMEM medium containing $10 \%$ FBS and $1 \%$ L-Gln, the cell culture was incubated at $37^{\circ} \mathrm{C}$ in a $5 \% \mathrm{CO}_{2}$ atmosphere. The medium was changed, and the cells were passaged every $2-3$ days.

\section{Induced differentiation of BMSCs into osteoblasts, adipocytes and neuroblasts}

The second generation of BMSCs were divided into control and induction groups. Induced differentiation of BMSCs into osteoblast cells (short for DBOCs) was conducted by culturing in a complete culture medium containing $10 \mathrm{mmol} / \mathrm{L} \beta$-glycerophosphate, $10^{-8} \mathrm{~mol} / \mathrm{L}$ dexamethasone and $50 \mathrm{mg} / \mathrm{mL}$ Vitamin C (Amresco USA). The cells were cultured in this medium for 21 days, then an alkaline phosphatase (ALP) assay was performed to identify the differentiated cells in the medium (17). Induced differentiation of BMSCs into adipocyte cells (short for DBACs) was conducted by using the complete culture medium containing $10^{-6} \mathrm{~mol} / \mathrm{L}$ dexamethasone, $0.25 \mathrm{mg} / \mathrm{L}$ 
insulin and $0.05 \mathrm{mmol} / \mathrm{L}$ indomethacin (Amresco USA). The cells were cultured for 18 days, then the Oil red O (Amresco USA) staining method was used to identify the differentiated adipocytes in the medium (18). Finally, differentiation of BMSCs into nerve-like cells (short for DBNCs) was induced by culturing in the complete culture medium containing $1 \mathrm{mmol} / \mathrm{L} \beta$-mercaptoethanol. After maintaining the cell culture for 3 days, the medium was replaced with a serumfree medium and the cells were further cultured for another 3 days. An immunohistochemistry assay was performed to detect the neuron-specific enolase (NSE), which indicates the presence of nerve cells in the culture (19).

\section{Interruption of the induced differentiation of BMSCs into osteoblasts, adipocytes and neuroblasts}

Induced differentiation of BMSCs into osteoblasts, adipocytes and neuroblasts was interrupted by a media change. The BMSC differentiation for inducing the DBOCs was interrupted at the different stages by discarding the induction media on days $1,4,7$, and 10.5 . The adherent cells were washed three times with a $0.01 \mathrm{M}$ PBS, then they were cultured in the complete medium until day 21 . In the course of inducing the DBACs, the BMSC differentiation culture was interrupted at different stages by discarding the induction media on days 1, 3, 6 and 9. The adherent cells were washed 3 times with $0.01 \mathrm{M}$ PBS, then they were cultured in the complete medium until day 18 . In the course of inducing the DBNCs, the BMSC differentiation culture was interrupted at different stages by discarding the induction media at 24, 44, 56 and 72 hours. The adherent cells were washed 3 times with a $0.01 \mathrm{M}$ PBS, then they were cultured in the complete medium until day 6 .

\section{Soft agar colony formation experiment}

Low melting point agarose (1.2\%) (Bioworld, China) solution was prepared in triple distilled water. This solution was autoclaved and cooled to $37^{\circ} \mathrm{C}$. Then it was mixed homogenously at $37^{\circ} \mathrm{C}$ with an equal volume of $2 \times$ DMEM complete medium, which contained 20\% FBS. Finally, the bottom agar layers were prepared by pouring this solution mixture into the cell culture plates. These plates were ready for use once the agarose gel solidified and cooled down to room temperature. Another $0.7 \%$ low melting point agarose solution was also prepared using triple distilled water. This solution was autoclaved and cooled to $37^{\circ} \mathrm{C}$. Cell culture suspensions with a density of $1 \times 10^{3}$ cells $/ \mathrm{mL}$ were prepared in a $2 \times$ DMEM medium for all 4 cell groups: induced differentiation group, interrupted group, negative control and positive control. The cell culture suspensions were mixed with an equal volume of $0.7 \%$ low melting agarose solution. Finally, they were poured onto the $1.2 \%$ soft agar solution to form a double agar layer. In the experiments, BMSCs and Hela cells were used as the negative and positive control groups respectively. The cells were cultured for 15 days at $37{ }^{\circ} \mathrm{C}$ in an incubator with $5 \%$ $\mathrm{CO}_{2}$ atmosphere (20). The colony formation rates were calculated using the following formula:

$$
\text { Colony formation rate }=\frac{\text { number of clones }}{\text { number of inoculated cells }} \times 100
$$

\section{Animal tumorigenesis experiment}

The selected clones from the soft agar gel were picked and cut into small pieces using sterile razor blades. These pieces were inoculated into a fresh DMEM/F12 medium. The clones were cultured in cell culture flasks for 3 days. The culture medium was discarded after 3 days, and the adherent cells were digested with $0.25 \%$ trypsin. The trypsinized cells were suspended in a small amount of a DMEM/F12 medium. After this, they were transferred into a new cell culture flask containing DMEM/F12 medium. These cells were cultured in the medium for 5 days, and, in this way, the soft agar cells were successfully enriched. The cells from the interrupted group, the negative control (BMSCs) group and the positive control (Hela cells) group were also obtained and the suspensions of these cells in a DMEM medium were prepared. Each cell suspension had a density of $2 \times 10^{7}$ cells $/ \mathrm{mL}$. All the 3 suspensions were stored at $4^{\circ} \mathrm{C}$.

Twenty-four hours before the experiment the cyclophosphamide (CTX)-immunosuppressed mice were prepared by subcutaneously injecting a $200 \mathrm{mg} / \mathrm{kg}$ CTX (Bioworld, China) in 75 Kunming mice. Before inoculation, the CTX immunosuppressed mice were anesthetized for 15-30 min, then surgery was conducted to open the back of each mouse and $0.2 \mathrm{~mL}$ of the requisite cell suspension was injected into one side of the renal fascia (21). The anatomy examinations were carried out to inspect the resultant tumor on days 7, 10, 14, 17 and 20 after surgery and inoculation.

\section{Identification of cell morphology}

Wright-Giemsa stain and Hemotoxylin-eosin (HE) stain were purchased from Zhuhai Beisuo Biotechnology Co. 

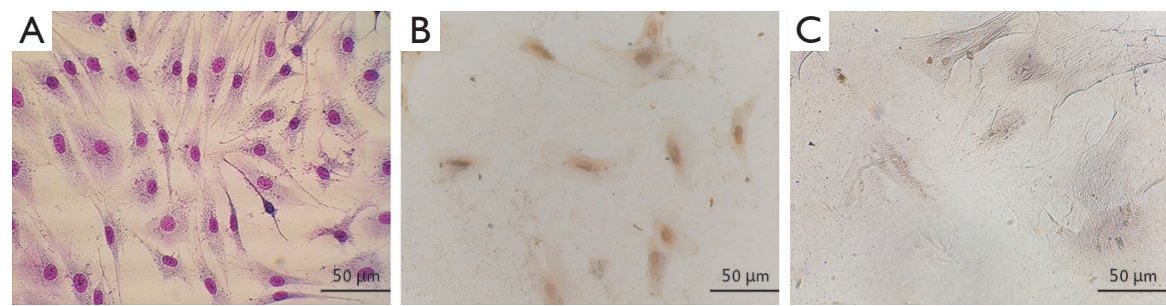

Figure 1 Identification of the BMSCs characteristics. (A) Wrights-Giemsa staining of the BMSCs; (B) the immunohistochemical staining of the CD44 antibody; (C) the immunohistochemical staining of the CD34 antibody.

Ltd. (Zhuhai, China), and the staining procedures were performed as per manufacturer's instructions.

\section{Immunobistochemistry}

Immunohistochemical staining procedures were performed as per manufacturer's instructions. All antibodies were purchased from Beijing Biosynthesis Biotechnology Co., Ltd. (Beijing, China) and diluted as follows: CD34 (1:200), CD44 (1:200), NSE (1:200), Bone morphogenetic protein 4 (BMP4) (1:300), S-100 (1:300), Nestin (1:300) and CD133 (1:300).

\section{Results}

\section{Induced differentiation of BMSCs into osteoblasts, adipocytes and neuroblasts isolation and culture of BMSCs}

After the bone marrow cell suspension was prepared it was cultured for 8 days. The cultured cells were passaged six times at a cell density of $3 \times 10^{4}$ cells $/ \mathrm{mL}$. The WrightGiemsa staining of all the cells were adherent and most of them were spindle-shaped in their appearance. They had full cell bodies, clear contours, and grew in a spiral or comb pattern (Figure 1A). The cells showed a healthy morphology and high proliferative activity. Immunohistochemical staining confirmed the cell surface markers of CD44 and CD34. The results showed that the BMSCs marker CD44 was positive in the cytoplasm (Figure 1B), but the hematopoietic stem cell surface marker CD34 was negative (Figure 1C), demonstrating typical surface markers of BMSCs.

\section{Induced differentiation of BMSCs}

After culturing the DBOC differentiation cells for 21 days, the Wright-Giemsa staining of the cells (Figure
$2 A$ ) showed triangular or polygonal cells with large, round nuclei, and they underwent cell fusion to form dense mineralized nodules. Figure $2 B$ illustrates the findings of the ALP activity assay: large brown-black particles or massive precipitates were observed in the cytoplasm, indicating that BMSCs had differentiated into a mature osteoblast.

After culturing the DBAC differentiation cells for 18 days, the Wright-Giemsa staining (Figure 2C) showed that there were round or polygonal cells with enlarged cytoplasm, and lipid droplets were also visible in the cytoplasm. As shown in Figure 2D, the oil red O staining revealed that the cells were filled with orange-red lipid droplets, which appeared like strings of beads. The nuclei were pushed aside by these big lipid droplets. The results clearly indicate that the BMSCs had differentiated into mature adipocytes.

In this experiment, the DBNC differentiation cells were cultured for 72 hours, and an additional 3 days culture in serum-free medium. As shown in Figure 2E, WrightsGiemsa staining of cell culture revealed that the cells had bipolar or complex multipolar morphology, had developed branches to form pseudopodia, and the cytoplasm gradually shrunk toward the nucleus. Some of the adjacent cells had also formed a net like structure. As shown in Figure $2 \mathrm{~F}$, NSE immunohistochemistry staining revealed that the cell bodies were brownish-yellow in color. This indicates that the BMSCs had differentiated into mature neurons.

In the above three groups of experiments, different inducing conditions were used to successfully induce the BMSCs to differentiate into osteoblasts, adipocytes and neurons, indicating that the initial BMSCs were pluripotent in nature.

\section{Interrupted differentiation into DBOCs, DBACs and DBNCs}

Stem cells follow a specific differentiation process, and they eventually form mature cells that do not differentiate. We 

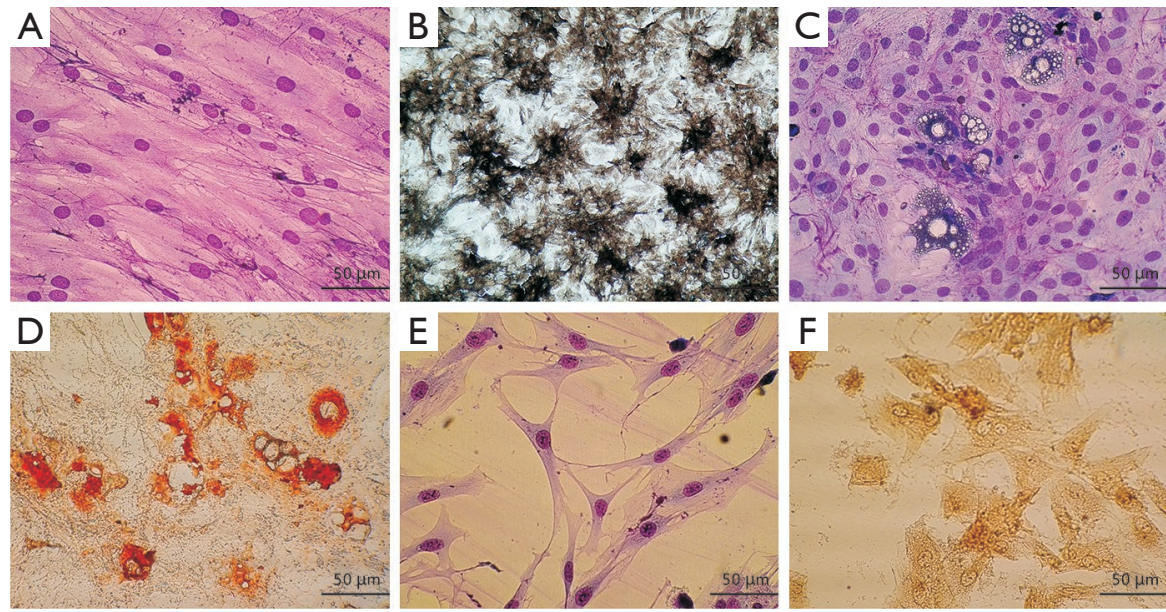

Figure 2 Identification of the cell characteristics of the induced BMSCs differentiating into osteoblasts, adipocytes, and neuroblasts. (A) The Wrights-Giemsa staining of the DBOC differentiation cells for 21 days; (B) the expression of ALP in the DBOC differentiation cells for 21 days; (C) the Wrights-Giemsa staining of the DBAC differentiation cells for 18 days; (D) the oil red O staining of the DBAC differentiation cells for 18 days; (E) the Wrights-Giemsa staining of the DBNC differentiation cells were cultured for 72 hours, and an additional 3 days culture in serum-free medium; (F) the expression of NSE in the DBNC differentiation cells were cultured for 72 hours, and an additional 3 days culture in serum-free medium.

established stem cell models of interrupted differentiation by discarding and replacing the inducing media at different time intervals in the course of the differentiation culture. At the same time a variety of biochemical and immunohistochemical assays were conducted including WrightGiemsa staining, ALP activity assay, oil red $\mathrm{O}$ staining and NSE immuno-histochemical staining to assess the extent of induced differentiation of BMSCs into osteoblasts, adipocytes and neuroblasts when the interruption process was imposed.

Figure $3 A, B, C, D, E, F$ shows the ALP activity of the cells undergoing induced differentiation toward DBOCs. Almost no ALP activity was observed in the negative control cell group, however, in the cells undergoing a differentiation process of the DBOCs, 1 day after induction culture, a very small amount of the brownish-black plaques appeared in the cytoplasm of cells, indicating an extremely low ALP expression. The number of plaques increased steadily in the DBOC differentiation cells on days 4 to 7 of the differentiation, indicating that the ALP expression increased as the differentiation progressed. The plaques seen in the DBOC differentiation cells on day 10.5 were significantly darker than the previous ones, indicating a higher ALP expression in the DBOCs obtained on day 10.5. The ALP expression reached maximal levels on day 21 during the differentiation culture, and a large amount of the brownish black plaques and massive precipitates in the cell cytoplasm of DBOCs were observed.

Figure $3 G, H, I, \mathcal{F}, K, L$ shows the oil red $\mathrm{O}$ staining results of the cells undergoing induced DBAC differentiation. In the negative control group of BMSCs, no lipid droplets in the cytoplasm were observed. Even in the DBAC induction cells on day 1 and 3 during differentiation, no significant lipid droplets were generated in the cytoplasm. The cells subject to interrupted differentiation of the DBACs on day 6 showed tiny droplets in the cytoplasm and on day 9 the lipid droplets had attained a uniform size in the cytoplasm. Large lipid droplets in the cytoplasm of the DBAC differentiation cells formed after 18 days of differentiation culture. The lipid droplets were orange-red in color and appeared like strings of beads in the cytoplasm of DBACs.

Figure $3 M, N, O, P, Q, R$ illustrates NSE immunohistochemical staining results of the interrupted DBNC differentiation cells. There was no expression of NSE in the cytoplasm of the BMSCs, which constituted the negative control group. Low expression of NSE was found in the DBNC differentiation cell group after 24 and 44 hours of induced differentiation. The expression of NSE was elevated in the cells obtained after 56 hours of induced differentiation for DBNC. Moreover, the expression of NSE increased significantly in the DBNC differentiation cells obtained after 72 hours of induced differentiation. 


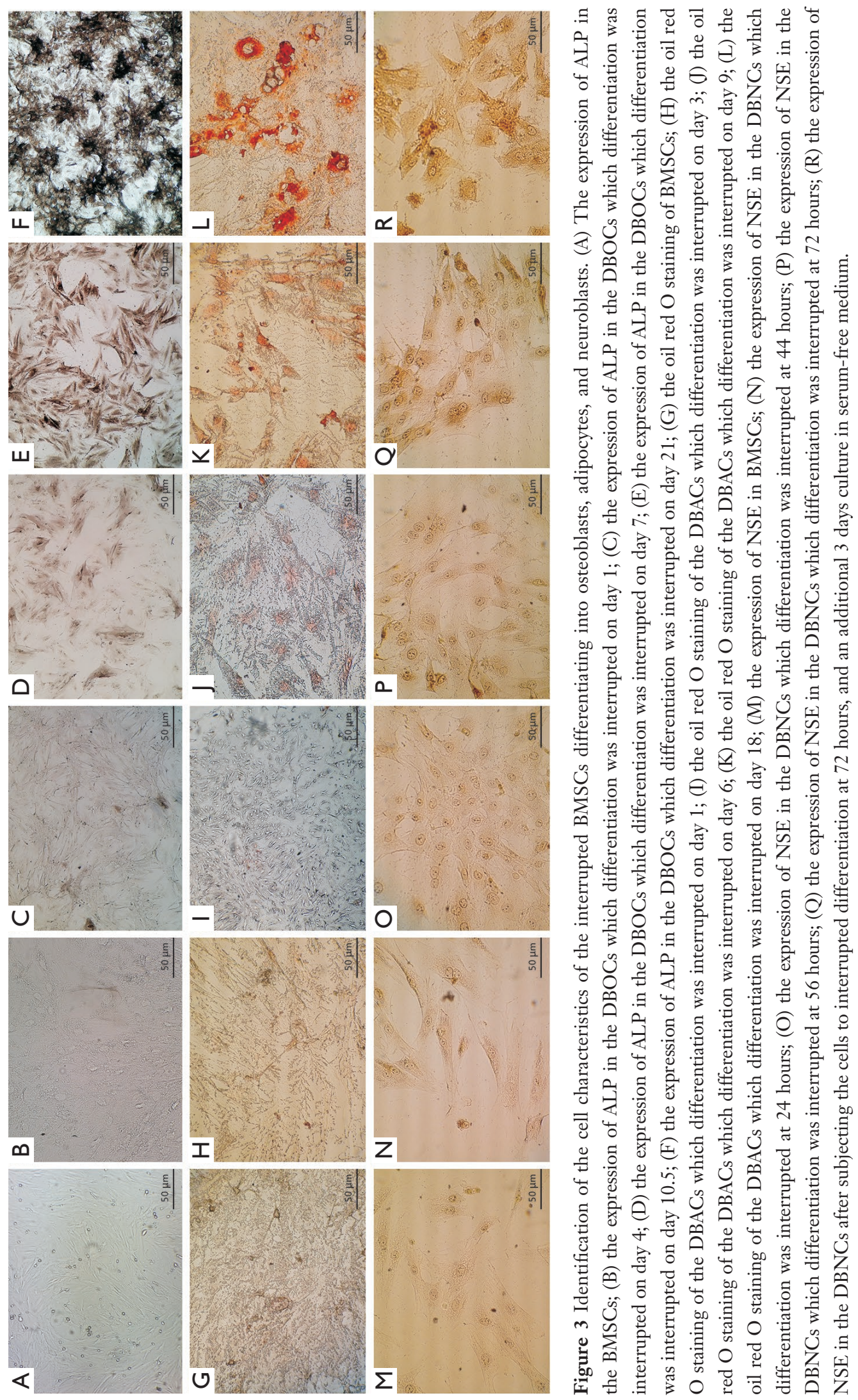



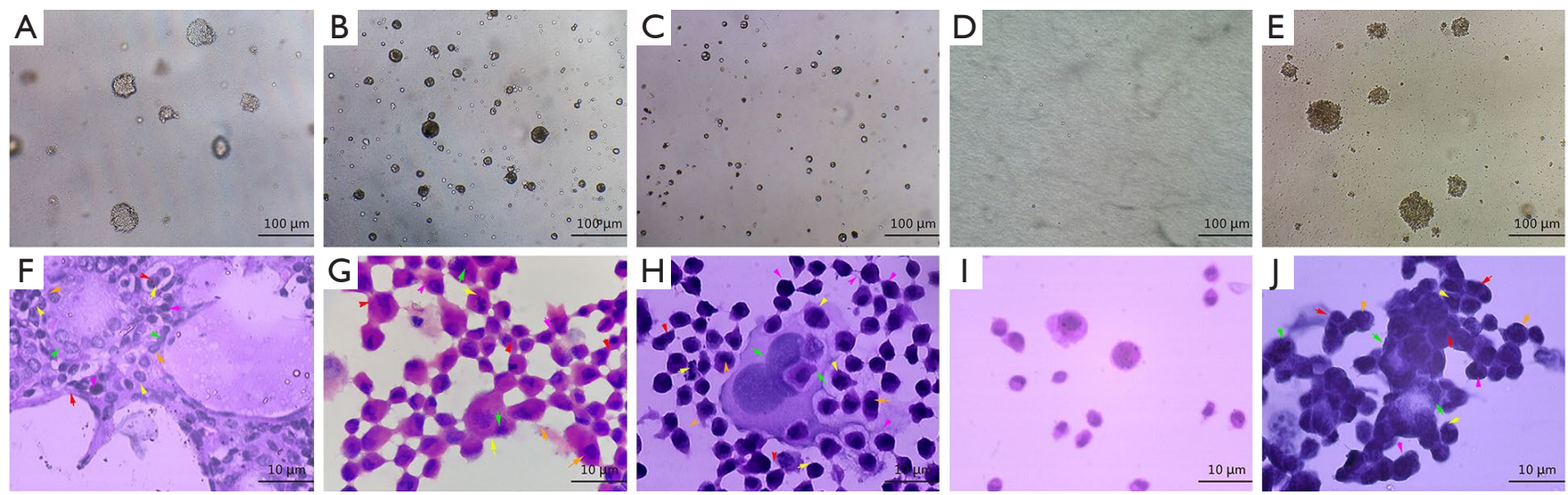

Figure 4 Soft agar colony formation and HE staining for morphological identification of clonal cells. (A) The DBOCs in soft agar (from the cells interrupted on day 4); (B) the DBACs in soft agar (from the cells interrupted on day 3); (C) the DBNCs in soft agar (from the cells interrupted at 44 hours); (D) the BMSCs in soft agar; (E) Hela cells in soft agar; (F) HE staining of the enriched DBOCs in soft agar (from the cells interrupted on day 4); (G) HE staining of the enriched DBACs in soft agar (from the cells interrupted on day 3); (H) HE staining of the enriched DBNCs in soft agar (from the cells interrupted at 44 hours); (I) HE staining of the BMSCs; (J) HE staining of the enriched Hela cells in soft agar. Morphological abnormalities are indicated by colored arrows: nuclear fission, red; multi-nucleation, yellow; phagocytosis, green; changes in the cytoplasm-nuclei-relation, orange; large nuclei and deep staining, pink.

These DBNCs were cultured for 3 days in a serum-free medium and the expression of NSE was the highest and developed clear brownish yellow color in the cytoplasm of DBNCs.

When interruption of the differentiation occurred at time-point close to the cell maturation time, the characteristics of the differentiated cells would more closely resemble those of mature cells, while bearing very low cell proliferation ability. In our studies induced differentiation of BMSCs were interrupted at different time points in the course of differentiation, and we obtained the differentiated cells at different stages indicated by different levels of characteristic biochemical and immuno-histochemical staining parameters. These differentiated cells at different levels were transferred into the complete culture medium. We found that the differentiated cells had preserved their characteristics at the interruption time point and maintained the same proliferation rate of the culture in the passages.

\section{Soft agar colony formation experiment}

Contrary to normal cells, it is well established that tumor stem cells or cancer cells do not have a contact inhibition in any medium and can penetrate through the semi-solid medium, and therefore are able to keep proliferating and form colonies in a semi-solid medium (22). In this study, the negative control group consisted of BMSCs, while the positive control group consisted of Hela cells. The differentiation interrupted group consisted of cells obtained in the course of interrupted differentiation of BMSCs. Soft agar colony formation experiments were performed with the cells of all the three groups. As shown in Figure 4A,B,C,D,E and Table 1, the colony formation ability of the interrupted differentiation cells for DBOCs, DBACs and DBNCs was shown to be the strongest when the interruption was conducted on day 4, day 3 and at 44 hours respectively, during the course of differentiation. Hela cells showed the highest potency of colony-forming. Colony forming cells were isolated from the soft agar medium and cultured for another 5 days. All the cells obtained from the interrupted differentiation of the BMSCs and showing a colony formation demonstrated vigorous growth in the following culture; they all featured a loss of contact inhibition, colony formation and strong proliferation. HE staining with these cells was conducted and it was observed that the cells had the characteristic features of malformed cells, such as a nuclear fission, multi-nucleation, phagocytosis, changes in the cytoplasm-nuclei-relation, large nuclei and deep staining (Figure 4F,G,H). On the other hand, there was no significant nuclear abnormality in BMSCs (Figure 4I). However, Hela cells displayed typical cancer cell characteristics, such as significant cell heterogeneity, large nuclei, deep staining 
Table 1 Soft agar clone formation rate

\begin{tabular}{lc}
\hline Interruption cells & $\begin{array}{c}\text { Soft agar clone } \\
\text { formation rate }\end{array}$ \\
\hline Negative control BMSCs group & $0 \%$ \\
Positive control Hela cell group & $9.1 \%$ \\
Interruption of DBOCs for 1 day & $0.31 \%$ \\
Interruption of DBOCs for 4 days & $7.8 \%$ \\
Interruption of DBOCs for 7 days & $0.25 \%$ \\
Interruption of DBOCs for 10.5 days & $0 \%$ \\
Interruption of DBOCs for 21 days & $0 \%$ \\
Interruption of DBACs for 1 day & $0.71 \%$ \\
Interruption of DBACs for 3 days & $7.19 \%$ \\
Interruption of DBACs for 6 days & $0.64 \%$ \\
Interruption of DBACs for 9 days & $0 \%$ \\
Interruption of DBACs for 18 days & $0 \%$ \\
Interruption of DBNCs for 24 hours & $0.42 \%$ \\
Interruption of DBNCs for 44 hours & $6.89 \%$ \\
Interruption of DBNCs for 56 hours & $0.37 \%$ \\
Interruption of DBNCs for 72 hours & $0 \%$ \\
Interruption of DBNCs for 24 hours and & $0 \%$ \\
\hline
\end{tabular}

and increased nuclear-cytoplasmic ratio (Figure 4f). These results suggested that the cells obtained from interrupted differentiation of BMSCs tend to abnormal evolution including loss of contact inhibition, high proliferation and cancerous cellular changes.

\section{Animal tumorigenesis experiment with BMSCs undergoing interrupted differentiation}

It was noticeable that partial stem cells after an interrupted differentiation acquired colony forming abilities, high proliferation and tend to have an abnormal evolution. It would be interesting to further investigate whether these cells may be necessarily tumorigenic in nature. Animal tumorigenesis experiments with the cells of all the above mentioned three groups were performed. The differentiation interrupted cells were screened and isolated from the soft agar experiment along with the Hela cells. The negative control BMSCs were prepared as cell suspensions with a density of approximately $2 \times 10^{7}$ living
cells/mL for animal tumorigenesis experiments.

The cells of DBOC group on which interruption was conducted on the fourth day of differentiation were used to inoculate the mice. After 9 days of surgery, white, hard and enlarged hyperplasia was observed in the kidney; the lower part of the kidney showed adhesion to some soft tissues that were white in color. It was also noticeable that an abnormally enlarged spleen was severely adhesive to the kidney. By performing HE staining of the paraffin sections, the cells showed irregular shapes and sizes, significantly large and deeply stained nuclei, and a high nuclear-cytoplasmic ratio. These cells were particularly spread around the pancreatic tissue. There was significant necrosis in the central part of the connection between the pancreas and the kidney. The concentration of cancer cells was greater around the necrotic area. Pathological mitoses were also observed in cancer tissues. Immunohistochemical staining results showed the existence of BMP4 in the cytoplasm. Moreover, the cancer stem cell markers CD133 in the cytoplasm and cell membrane of the cells were also detected (Figure $5 A, B, C, D$ ).

The cells of DBAC group on which interruption was conducted during the third day of the differentiation, were used to inoculate the mice for the animal tumorigenesis experiment. 14 days after inoculation, white hyperplasia was observed at the inoculation sites of the renal fascia, which were adhesive to some soft tissues of white color. HE staining of the paraffin sections revealed several irregularly sized tumor cells scattering around the kidney, with large nuclei and deep staining. The central part of the tumor tissue showed necrosis. Slight pathological mitoses in the tumor cells were also observed, so it was diagnosed as cancer tissue. Immunohistochemical staining detected the adipocyte marker S-100B in the cytoplasm. In addition, it was also observed that there was cancer stem cell marker CD133 in the cytoplasm and cell membrane of the cells (Figure $5 E, F, G, H)$.

The cells of DBNC group on which interruption was conducted after 44 hours of differentiation were used to inoculate the mice for the animal tumorigenesis experiment. 14 days after inoculation, the anatomy of the inoculated mice showed that although both the kidneys were swollen, only the latter kidney appeared hard and white in color. There was a significantly abnormal proliferation of white mass in the kidney, and a soft white tissue had completely covered the renal fascia. HE staining results of the paraffin sections revealed that the necrosis was significant around the renal fascia. Moreover, a large number of irregular 

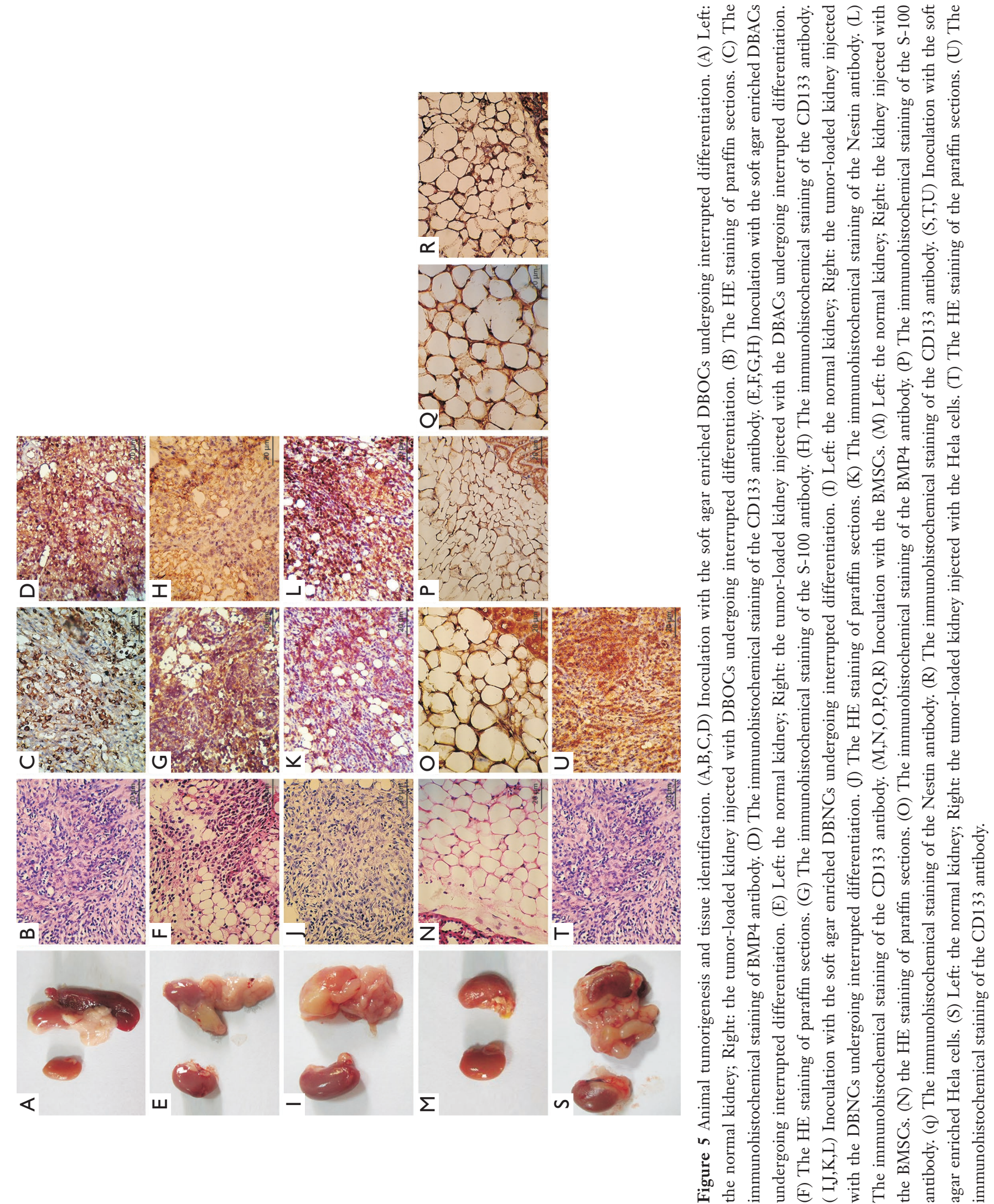


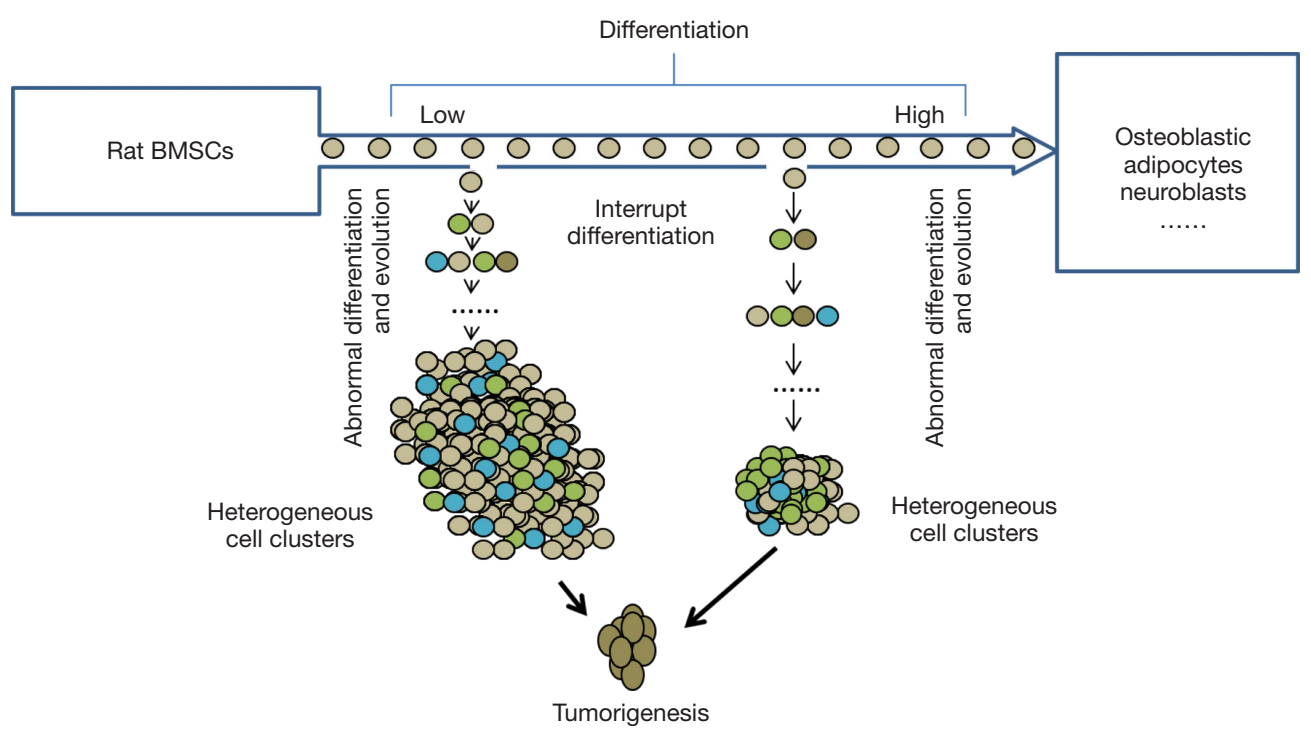

Figure 6 Schematic diagram for the abnormal differentiation and evolution of rat BMSCs and formation of tumor.

tumor cells had grown around the necrotic area, and these tumor cells had invaded the normal kidney tissue. Multiple pathological mitoses were observed in the tumor tissues, so they were diagnosed as cancer tissues. Immunohistochemical staining detected the neural stem cell marker Nestin in the cell membrane. In addition, cancer stem cell marker CD133 in the cytoplasm and the cell membrane was also detected (Figure 5I, $\mathcal{F}, K, L)$.

\section{Animal tumorigenesis experiment for BMSCs and Hela cells}

In the mice inoculated with BMSCs, both the kidneys appeared normal as they did not develop any abnormalities. This was confirmed by examining the anatomical picture and the paraffin sections of both the kidneys of these inoculated mice. Immunohistochemical staining did not find BMP4, S-100B, Nestin or any other tumor stem cell markers in the cells (Figure $5 M, N, O, P, Q, R$ ). In the mice inoculated with Hela cells, anatomical examination of the renal fascia showed that soft, white tissues were adhering and covering the normal renal tissues like a ball, indicating the unique adhesion properties of cancer cells. By performing HE staining of the paraffin sections, phagocytosis in the adipose tissues surrounding the kidney was observed, which was itself also being squeezed by the concentrated tumor cells. These cells had greater nuclear-cytoplasmic ratios and higher necrosis in the central parts. A clear boundary was visible between the necrotic tissue and the surrounding immune cells, such as neutrophils and lymphocytes. Because pathological mitoses appeared in the necrotic tissue, it was diagnosed as cancer tissue. Immunohistochemical staining revealed high levels of CD133 in the cytoplasm and cell membrane (Figure 5S,T,U). The above results also indicated that most inoculated cells died out in the microenvironment of renal fascia, although few cells evolved into cancerous tissue.

\section{Discussion}

Our studies provide new insight into the pathogenesis of tumors in respect to stem cells. The results primarily proved that tumors occur when the somatic stem cells (SSC) evolve and differentiate abnormally into heterogeneous cell clusters (Figure 6).

Previous studies have made great progress in comprehending the evolution of the cancer genome and have elaborately explained the dynamic interplay between tumor cells and the microenvironment. However, scientists have barely been able to elucidate the mechanism of cancer (23-25), and the following aspects for the mechanisms of cancer still remain unclear: the pathogenesis of tumor stem cells, the mode of tumor evolution and the mechanisms for tumor metastasis in the microenvironment $(26,27)$. In our experiments, the differentiation inducers were removed at certain stipulated time points, thereby interrupting the induced differentiation of stem cells at various stages. Thus, 


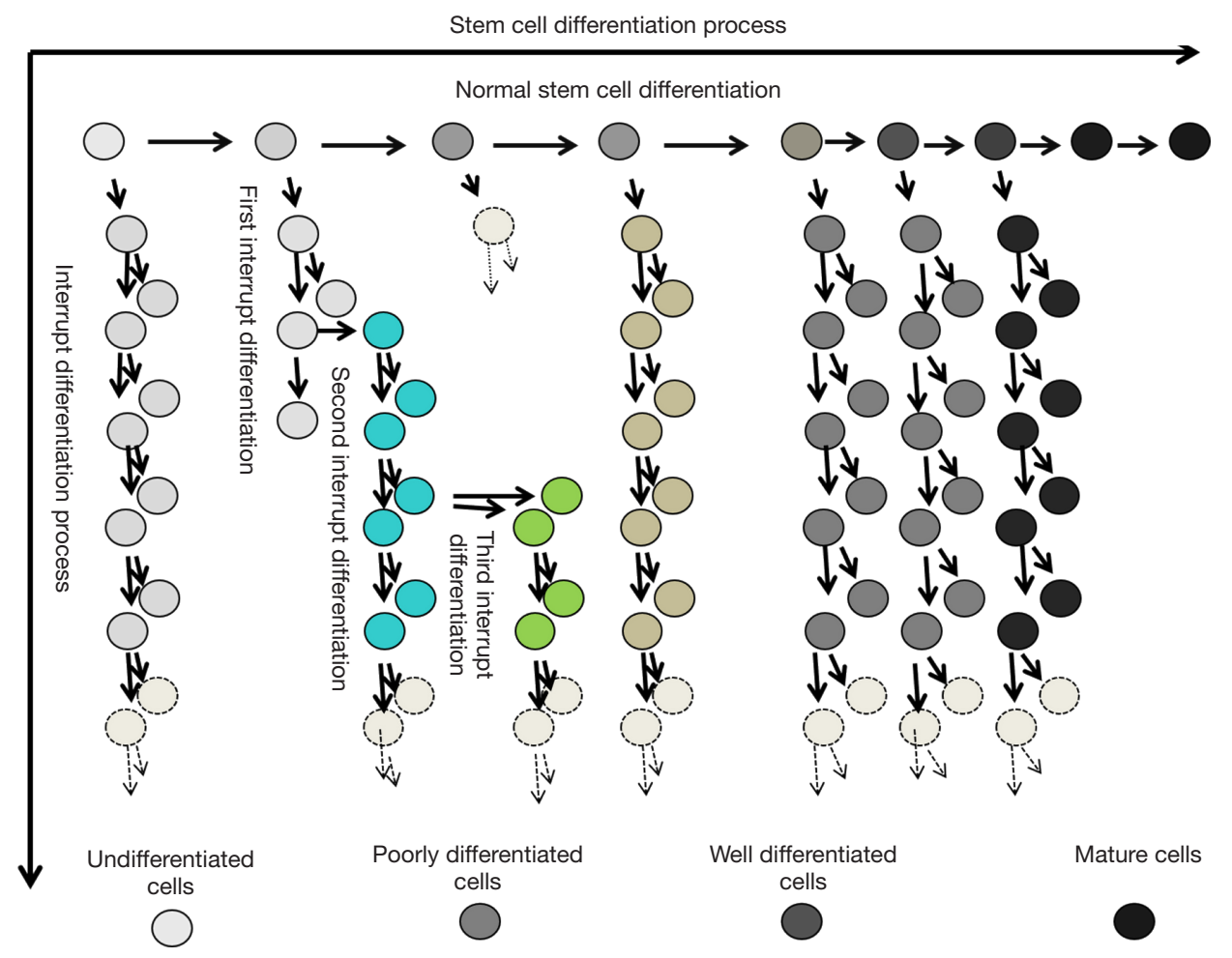

Figure 7 Schematic diagram for the stem cell differentiation process.

in a new microenvironment, the stem cells differentiated and evolved (Figure 7) into heterogeneous cell clusters and the outcome was further investigated. The soft agar colony formation experiments with the cells undergoing differentiation interruption demonstrated that cancer stem cells do exist in heterogeneous cell clusters. Moreover, the mice tumorigenesis experiments confirmed the tumorigenic ability of these cell clusters.

It is worthwhile to mention that SSC are undifferentiated stem cells that exist in all differentiated tissues, and that these cells have the ability to differentiate and overcome their "developmental limitations". They can even induce the cross lineage and ectoderm to differentiate into other tissue types. In a specific area of the tissue, SSC can exist in a dormant state for years. In general, they become active only when the tissue is injured or diseased. In other words, the totipotency of SSC is similar to that of embryonic stem cells $(28,29)$. In our studies, BMSCs were chosen because they are well investigated in most studies of SSC, and BMSCs have the highest maturity among all types of SSC.

Stem cell differentiation is the process through which cells of the same origin gradually transform into cell populations of different morphologies, structures and functions. Stem cells get activated when an abnormality develops in the cells and/or microenvironment; however, stem cells may stop differentiating at a certain point. At this point, stem cells can still proliferate but not differentiate, leading to the formation of heterogeneous cell clusters. There are numerous ways to interrupt the induced differentiation of stem cells. In this experiment, we used the strategy of replacing the induction medium with the normal medium, but we did not add any blocking agent. Thus, we ensured that the interruption was effective and accurate.

In molecular biology, stem cell differentiation is due to the selective expression of the cell genome in a small timespace window. Therefore, the process of differentiation can be controlled, and even cells of a different origins and lines can be formed by simply turning on and off the expression of different genes. When the stem cell differentiation is interrupted, only the gene expression promoted by the specific inducer stops; however, the cells continue to be multipotent. In a new microenvironment, a different inducer may be used to trigger the stem cells deviating from the original differentiation pathway and evolving into the heterogeneous cell clusters. These clusters adapt easily with the new environment (30). Cellular evolution refers to the 
Table 2 Yielding types of the heterogeneous cell clusters

\begin{tabular}{|c|c|c|c|c|c|c|c|c|}
\hline $\begin{array}{l}\text { Heterogeneous } \\
\text { cell cluster type }\end{array}$ & \multicolumn{2}{|c|}{ The first time } & \multicolumn{2}{|c|}{ The second times } & \multicolumn{2}{|c|}{ The $\mathrm{N}$ times } & Evolution & Result \\
\hline 1 & Interrupt & $\begin{array}{l}\text { Sustained } \\
\text { proliferation }\end{array}$ & $\begin{array}{c}\text { Not } \\
\text { happening }\end{array}$ & $\begin{array}{c}\text { Not } \\
\text { happening }\end{array}$ & $\begin{array}{c}\text { Not } \\
\text { happening }\end{array}$ & $\begin{array}{c}\text { Not } \\
\text { happening }\end{array}$ & $\begin{array}{c}\text { Not } \\
\text { happening }\end{array}$ & $\begin{array}{l}\text { Heterogeneous } \\
\text { cell cluster }\end{array}$ \\
\hline 2 & Interrupt & $\begin{array}{c}\text { Persistent } \\
\text { proliferation and } \\
\text { genetic mutation }\end{array}$ & $\begin{array}{c}\text { Not } \\
\text { happening }\end{array}$ & $\begin{array}{c}\text { Not } \\
\text { happening }\end{array}$ & $\begin{array}{c}\text { Not } \\
\text { happening }\end{array}$ & $\begin{array}{c}\text { Not } \\
\text { happening }\end{array}$ & $\begin{array}{l}\text { Evolution } \\
\text { of genetic } \\
\text { variation }\end{array}$ & $\begin{array}{l}\text { Heterogeneous } \\
\text { cell cluster }\end{array}$ \\
\hline 3 & Interrupt & - & $\begin{array}{c}\text { New } \\
\text { differentiation }\end{array}$ & $\begin{array}{l}\text { New } \\
\text { proliferation } \\
\text { rate }\end{array}$ & $\begin{array}{c}\text { Not } \\
\text { happening }\end{array}$ & $\begin{array}{c}\text { Not } \\
\text { happening }\end{array}$ & $\begin{array}{l}\text { Differential } \\
\text { evolution }\end{array}$ & $\begin{array}{l}\text { Heterogeneous } \\
\text { cell cluster }\end{array}$ \\
\hline 4 & Interrupt & - & $\begin{array}{c}\text { New } \\
\text { differentiation }\end{array}$ & $\begin{array}{c}\text { Persistent } \\
\text { proliferation } \\
\text { and genetic } \\
\text { mutation }\end{array}$ & $\begin{array}{l}\text { Multiple } \\
\text { differential } \\
\text { evolution }\end{array}$ & $\begin{array}{l}\text { Multiple } \\
\text { genetic } \\
\text { mutation }\end{array}$ & $\begin{array}{l}\text { Evolution of } \\
\text { differentiation } \\
\text { and genetic } \\
\text { variation }\end{array}$ & $\begin{array}{l}\text { Heterogeneous } \\
\text { cell cluster }\end{array}$ \\
\hline
\end{tabular}

survival of the fittest cells when subjected to cytogenetic, phenotypic and/or genotypic variations. In our experiments, the complete medium was used twice to effectively simulate the in-situ evolution of stem cells after the interruption of induced differentiation.

As shown in Table 2, there are four possible mechanisms for stem cells to differentiate into abnormal cells and forming heterogeneous clusters: (I) induced differentiation is interrupted but the cells continue to proliferate; (II) induced differentiation is interrupted, but the cells undergo genetic mutation and continue to proliferate; (III) induced differentiation is interrupted with the help of a new inducer, and the stem cells deviate from the original differentiation pathway to adapt with the microenvironment; and (IV) induced differentiation is interrupted due to a new inducer and a genetic mutation, but the cells adapt with the microenvironment.

This implies that the heterogeneous cell clusters possibly have the following characteristics: (I) histological similarity with cancer tissue sections, (II) sustained proliferation abilities, (III) the ability to mutate and to diversify under micro-environmental stress, (IV) histocompatibility with autologous cells and $(\mathrm{V})$ the ability to synergize with the surrounding cells of the microenvironment.

Comparison of the characteristics of cancer cells and heterogeneous cell clusters also reveals more detailed information about the evolution of cancer. Recently, Hanahan et al. summarized the ten hallmarks of human cancer formation in the study "Hallmarks of cancer: the next generation" (31). Some of those characteristics are applicable to the heterogeneous cell clusters. The heterogeneous cell clusters show a sustained proliferation, which is a characteristic that is quite similar to the ability of cancer cells in "sustaining proliferative signalling evading growth suppressors, resisting cell death, and enabling replicative immortality", and, are the same as the sustained proliferation of the heterogeneous cell clusters. Similarly, the cancer hallmark of "evading immune destruction" is akin to the histological compatibility of the heterogeneous cell clusters. While "metastasis" of cancer is the ability of cancer cells to invade different tissues, we may be able to define the metastatic property of heterogeneous cell clusters as the existence of SSC in all the differentiated tissues and their ability to generate new clusters with various microenvironment, activation factors, and inducers. In this work, we found that heterogeneous cells have many characteristics of stem cells, such as differentiation, regeneration ability and the ability to repair damaged tissues and organs by working synergistically with the surrounding stromal cells. So far, the tumorigenesis effect of these differentiation interrupted stem cells in humans remains unknown, and we will further investigate the effect of these stem cells on various characteristics of angiogenesis, including the creation of a tumor microenvironment, reprogramming of energy metabolism and the active invasion of cells.

\section{Conclusions}

In summary, we have proven that the heterogeneous cell 
clusters were formed by the abnormal differentiation and evolution of SSC, that these clusters have tumorigenic ability, and that they display the basic hallmarks of cancer cells. Therefore, we hypothesize that cancer tissues are basically heterogeneous cell clusters with sustained proliferation abilities, which are formed by the abnormal differentiation and the evolution of SSC.

\section{Acknowledgments}

We thank Yuanqiang Liu, Jun Zhou, and members of the Medical Molecular Lab of Medical Science Institute in Gansu, China for their helpful discussion and encourage. Funding: This work was supported by the National Natural Science Foundation of China (grant numbers: 30960104 and 81560346 to S Liao) and the Innovation Foundation of China and Research Exploitation item (grant numbers: GKSOU-B06-10, GKS031030).

\section{Footnote}

Conflicts of Interest: TAll authors have completed the ICMJE uniform disclosure form (available at http://dx.doi. org/10.21037/tcr.2018.12.16). The authors have no conflicts of interest to declare.

Ethical Statement: The authors are accountable for all aspects of the work in ensuring that questions related to the accuracy or integrity of any part of the work are appropriately investigated and resolved. The study was approved by the Animal Ethics Committee of Gansu University of Traditional Chinese Medicine (Lanzhou, China) (No. 2016-061) and conducted in accordance with the national and institutional guidelines for the use and care of animals.

Open Access Statement: This is an Open Access article distributed in accordance with the Creative Commons Attribution-NonCommercial-NoDerivs 4.0 International License (CC BY-NC-ND 4.0), which permits the noncommercial replication and distribution of the article with the strict proviso that no changes or edits are made and the original work is properly cited (including links to both the formal publication through the relevant DOI and the license). See: https://creativecommons.org/licenses/by-nc-nd/4.0/.

\section{References}

1. Nowell PC. The clonal evolution of tumor cell populations. Science 1976;194:23-8.

2. Maley CC, Reid BJ. Natural selection in neoplastic progression of Barrett's esophagus. Seminars in Cancer Biology 2005;15:474-83.

3. Anderson AR, Weaver AM, Cummings PT, et al. Tumor morphology and phenotypic evolution driven by selective pressure from the microenvironment. Cell 2006;127:905-15.

4. Merlo LM, Pepper JW, Reid BJ, et al. Cancer as an evolutionary and ecological process. Nat Rev Cancer 2006;6:924-35.

5. Hamburger AW, Salmon S. Primary bioassay of human tumor stem cells. Science 1977;197:461-3.

6. Bonnet D, Dick JE. Human acute myeloid leukemia is organized as a hierarchy that originates from a primitive hematopoietic cell. Nat Med 1997;3:730-7.

7. Al-Hajj M, Clarke MF. Self-renewal and solid tumor stem cells. Oncogene 2004;23:7274-82.

8. Singh SK, Hawkins C, Clarke ID, et al. Identification of human brain tumour initiating cells. Nature 2004;432:396-401.

9. Hope KJ, Jin L, Dick JE. Acute myeloid leukemia originates from a hierarchy of leukemic stem cell classes that differ in self-renewal capacity. Nat Immunol 2004;5:738-43.

10. Warner JK, Wang JC, Hope KJ, et al. Concepts of human leukemic development. Oncogene 2004;23:7164-77.

11. Friedenstein AJ, Chailakhjan RK, Lalykina KS. The development of fibroblast colonies in monolayer cultures of guinea-pig bone marrow and spleen cells. Cell Tissue Kinet 1970;3:393-403.

12. Dean $M$, Fojo T, Bates $S$. Tumour stem cells and drug resistance. Nat Rev Cancer 2005;5:275-84.

13. Pittenger MF, Mackay AM, Beck SC, et al. Multilineage potential of adult human mesenchymal stem cells. Science 1999;284:143-7.

14. Pierce GB. Relationship between differentiation and carcinogenesis. Journal of toxicology and environmental health 1977;2:1335-42.

15. Potter VR. Phenotypic diversity in experimental hepatomas: the concept of partially blocked ontogeny. The 10th Walter Hubert Lecture. Br J Cancer 1978;38:1-23.

16. Tropel P, Noël D, Platet N, et al. Isolation and characterisation of mesenchymal stem cells from adult mouse bone marrow. Exp Cell Res 2004;295:395-406.

17. Maniatopoulos C, Sodek J, Melcher AH. Bone formation in vitro by stromal cells obtained from bone marrow of young adult rats. Cell and Tissue Research 
1988;254:317-30.

18. Beresford JN, Bennett JH, Devlin C, et al. Evidence for an inverse relationship between the differentiation of adipocytic and osteogenic cells in rat marrow stromal cell cultures. J Cell Sci 1992;102:341-51.

19. Black IB, Woodbury D. Adult rat and human bone marrow stromal stem cells differentiate into neurons. Blood Cells Mol Dis 2001;27:632-6.

20. Ochs RL, Fensterer J, Ohori NP, et al. Evidence for the isolation, growth, and characterization of malignant cells in primary cultures of human tumors. In Vitro Cell Dev Biol Anim 2003;39:63-70.

21. Peláez B, Campillo JA, Lopez-Asenjo JA, et al. Cyclophosphamide induces the development of early myeloid cells suppressing tumor cell growth by a nitric oxide-dependent mechanism. J Immunol 2001;166:6608-15.

22. Horibata S, Vo TV, Subramanian V, et al. Utilization of the Soft Agar Colony Formation Assay to Identify Inhibitors of Tumorigenicity in Breast Cancer Cells. J Vis Exp 2015;2015:e52727.

23. Tomasetti C, Li L, Vogelstein B. Stem cell divisions, somatic mutations, cancer etiology, and cancer prevention. Science 2017;355:1330.

24. Bolli N, Avet-Loiseau H, Wedge DC, et al. Heterogeneity of genomic evolution and mutational profiles in multiple

Cite this article as: Yuan H, Liao S, Zeng J, Huang X, Zhang J, Zhao J, Wang Y, Zhang H, Wang X, Liao S, Tang W, Wei Z, Tian C, Yang R, Liu F, Guo H, Zhang Y. Abnormally differentiated bone marrow mesenchymal stem cells develop into tumors in rats. Transl Cancer Res 2018;7(6):1643-1656. doi: $10.21037 /$ tcr.2018.12.16 myeloma. Nat Commun 2014;5:2997.

25. Casey SC, Amedei A, Aquilano K, et al. Cancer prevention and therapy through the modulation of the tumor microenvironment. Seminars in cancer biology 2015;35:S199-S223.

26. Schulenburg A, Blatt K, Cerny-Reiterer S, et al. Cancer stem cells in basic science and in translational oncology: can we translate into clinical application? J Hematol Oncol 2015;8:16.

27. Tomasetti C, Vogelstein B, Parmigiani G. Half or more of the somatic mutations in cancers of self-renewing tissues originate prior to tumor initiation. Proc Natl Acad Sci U S A 2013;110:1999-2004.

28. Goodell MA, Nguyen H, Shroyer N. Somatic stem cell heterogeneity: diversity in the blood, skin and intestinal stem cell compartments. Nat Rev Mol Cell Biol 2015;16:299-309.

29. Biteau B, Hochmuth CE, Jasper H. Maintaining tissue homeostasis: dynamic control of somatic stem cell activity. Cell Stem Cell 2011;9:402-11.

30. McGranahan N, Swanton C. Clonal Heterogeneity and Tumor Evolution: Past, Present, and the Future. Cell 2017;168:613-28.

31. Hanahan D, Weinberg RA. Hallmarks of cancer: the next generation. Cell 2011;144:646-74. 